\title{
Criminologie
}

\section{Foucault et les sciences humaines, un rapport de biais : l'exemple de la sociologie du droit}

\section{Pierre Lascoume}

Volume 26, numéro 1, 1993

Michel Foucault et la (post) modernité

URI : https://id.erudit.org/iderudit/017329ar

DOI : https://doi.org/10.7202/017329ar

Aller au sommaire du numéro

\section{Éditeur(s)}

Les Presses de l'Université de Montréal

ISSN

0316-0041 (imprimé)

1492-1367 (numérique)

Découvrir la revue

Citer cet article

Lascoume, P. (1993). Foucault et les sciences humaines, un rapport de biais : l'exemple de la sociologie du droit. Criminologie, 26(1), 35-50.

https://doi.org/10.7202/017329ar
Résumé de l'article

This article expresses some ideas on the application of the methodological and epistemological principles, drawn from the concept of Michel Foucault, to legal sociology. In fact, Michel Foucault urged that his work be considered a tool-box where useful working instruments could be found. Among these, we find three types of instruments, conceptual, methodological and epistemological. This article discusses four epistemological principles taken from the work of Michel Foucault, namely (I) the break with anthro-pologism and with the cult of Man ; (2) rejection of the universals of thought; (3) the description of the paradigms for truth, understood as the conditions for true discourse, as defined in specific social formations ; (4) critical materialism. We also take into account a double principle of objectivication in the work of Foucault. The first of these principles acts at a more global level whereas the second operates analytically. We discuss how these epistemological principles and these objectivication forms guided us in our related sociological studies on the law. We also show how these studies were inspired by the work of Foucault, who, like De la gouvernementalité, were published after his death. 
This article expresses some ideas on the application of the methodological and epistemological principles, drawn from the concept of Michel Foucault, to legal sociology. In fact, Michel Foucault urged that his work be considered a tool-box where useful working instruments could be found. Among these, we find three types of instruments, conceptual, methodological and epistemological. This article discusses four epistemological principles taken from the work of Michel Foucault, namely (1) the break with anthropologism and with the cult of Man; (2) rejection of the universals of thought; (3) the description of the paradigms for truth, understood as the conditions for true discourse, as defined in specific social formations; (4) critical materialism. We also take into account a double principle of objectivication in the work of Foucault. The first of these principles acts at a more global level whereas the second operates analytically. We discuss how these epistemological principles and these objectivication forms guided us in our related sociological studies on the law. We also show how these studies were inspired by the work of Foucault, who, like De la gouvernementalitê, were published after his death.

La relation de Michel Foucault avec les sciences humaines est pour le moins paradoxale. Les termes de ce paradoxe sont en gros les suivants :

- Aujourd'hui, en France en particulier, Foucault est souvent mieux reçu et perçu dans le milieu des sciences humaines que dans celui de la philosophie, qui est sa discipline de référence. On observe l'utilisation de démarches et de concepts foucaldiens dans des travaux de sciences humaines de toutes sortes: histoire, sociologie, sciences de l'éducation, en urbanisme, en droit, etc., travaux qui suscitent chez la plupart des philosophes proches de Foucault un regard pour le moins condescendant.

- Par contre, Foucault a toujours eu des mots très durs pour ces savoirs positivistes, collés à une vision anthropologique du monde et toujours soupçonnés de contribuer au renforcement et à la légitimation du pouvoir disciplinaire. Pour Michel Foucault, les sciences humaines sont une production

1. L'auteur est chercheur attaché au Conseil national de la recherche scientifique/ Groupe d'analyse des politiques publiques, Université de Paris I, 13, rue du Four, 75006 Paris. 
archétypique du «bio-pouvoir». Depuis Histoire de la folie, sa critique ne s'est pas démentie à ce propos.

D'où la position difficile qui consiste, pour des chercheurs en sciences sociales, à aller prendre chez Michel Foucault des instruments de travail. C'est une entreprise périlleuse. Voilà pourquoi sans doute l'emprunt se résume souvent:

— soit à des objets de surface: «l'asile», «la sexualité»;

- soit à de la vulgate théorique: «l'enfermement», «la discipline», «l'aveu», ce qui revient à faire de Michel Foucault un théoricien du contrôle social ou de l'aliênation;

- soit, plus rarement, peut-être parce que c'est beaucoup plus difficile, à l'emprunt ou à l'appui sur ses positions, ses interrogations épistémologiques.

Alors y a-t-il un «bon » et un «mauvais » usage de Foucault? C'est une question qui n'a pas de sens, car la pensée de Michel Foucault est fondamentalement anti-dogmatique. On a pu dire qu'il était «un philosophe sans philosophie", disons un théoricien sans doctrine ou, mieux encore, non pas un épistémologue théorique, mais un épistémologue en acte. C'est sans doute pourquoi il s'est refusé à «faire école», avait en horreur les disciples et les zélotes et a toujours poussé ceux qui étaient en contact avec lui à mettre leur propre pensée à l'épreuve de leurs propres objets de recherche. Enfin, il a souvent répété que la parole émise, le texte écrit n'appartiennent pas à leurs auteurs. Et il considérait, pour lui-même, qu'il n'avait aucun droit de propriété sur ses travaux et que personne ne pouvait prétendre faire le gendarme à sa place.

Je pense que la liberté du lecteur doit être absolument respectée. Le discours est une réalité qui peut se transformer à l'infini. Ainsi celui qui écrit n'a pas le droit de donner des ordres au sujet de l'utilisation de ses écrits.

La question qui se pose à nous est celle de savoir quelle pratique avoir de ses travaux. S'approprier Michel Foucault, pourquoi pas mais comment? Tout ce que je puis faire ici, c'est essayer de clarifier les principes que j'ai pu dégager de mes observations sur les usages de Michel Foucault et de mes propres bricolages.

Je ferai ceci de deux façons. En traçant tout d'abord un cadre général qui sera le rappel du positionnement spécifique de Michel Foucault vis-à-vis des sciences humaines et nous donnera les éléments d'un cadre épistémologique. Puis je préciserai mon propos et $m$ 'exposerai davantage, 
en soulignant les apports que j'ai pu retirer de Michel Foucault pour ma pratique de la sociologie du droit.

Je me suis en effet servi de ses réflexions sur le pouvoir pour conceptualiser d'une certaine façon les activités juridiques :

- les activités de production des normes juridiques, c'est-à-dire les actions par le droit;

- mais aussi les activités de mobilisation et d'application du droit, c'est-à-dire le droit en action.

C'est à travers ces deux dimensions que je me suis efforcé de problématiser la dimension juridique des relations de pouvoir. Il s'agit d'un versant de la sociologie qui est encore peu développé et où dominent des problématiques classiques formulées en termes de légitimité ou d'effectivité des règles. Certains travaux de Michel Foucault m'ont beaucoup aidé à sortir des approches normative et morale du juridique. Comment alors penser le droit sans déboucher immanquablement sur une métaphysique du «bon droit» ou sur une pragmatique du «droit efficace»?

\section{LE BIAIS COMME TACTIQUE FOUCALDIENNE: POURQUOI ET COMMENT PENSER AUTREMENT?}

L'épistémologie se propose d'analyser les conditions de production et de validité des disciplines scientifiques. Foucault se rattache au courant qui envisage l'épistémologie comme une histoire conceptuelle des savoirs. Ses prédécesseurs, Koyre, Canguilhem, avaient pris comme terrain d'observation les sciences exactes. Foucault privilégia les sciences humaines et interrogea les vérités qu'elles énonçaient en les regardant de biais.

Chez Michel Foucault, le biais n'est pas un détour, un moyen artificiel pour atteindre un but. On doit prendre cette image à son sens premier, celui d'une oblique par rapport à une direction principale, d'une diagonale par rapport au droit fil.

\subsection{Le biais comme tactique intellectuelle}

Une des grandes questions de Foucault est celle des conditions de possibilité d'une pensée autre. Il semble sans cesse se demander comment il fut et comment il sera possible de sortir de la simple répétition mentale et de penser autrement. Pour sa part, il choisit un positionnement de biais qui traduit la recherche d'un déplacement, la mise en déséquilibre potentiellement créatif, qui initie un mouvement de la pensée et occasionne une 
pénétration inattendue. On peut ici utiliser la métaphore du prisme et du rayon de lumière: confronté de face à la surface de verre, le rayon éblouit tout. Pénétrant de biais, il se diffracte, révèle une texture particulière de la matière et surtout se révèle lui-même composite, fragmenté, pluriel.

On peut donner de ce positionnement foucaldien différentes illustrations:

- le plus typique est sans doute le premier chapitre de Les mots et les choses, intitulé «Les suivantes» (pp. 19-31), où Foucault explore les jeux de visibilité directe et indirecte utilisés par Vélasquez et d'où il tire une théorie de la représentation;

- également, dans L'archéologie du savoir, avec les dialogues qui achèvent l'introduction (p. 28) et forment la totalité de sa conclusion (pp. 259-275). Se décentrant par rapport à son propre discours, Foucault devient son propre critique et, anticipant sur le reproche d'être toujours en déplacement, il revendique la posture: «Je ne suis pas là où vous me guettez, mais ici d'où je vous regarde en riant";

- dans le cours inaugural au Collège de France, qui constitue le texte de L'ordre du discours, Foucault ne se positionne pas en «donneur de leçon» ni en maître dont la parole se substitue désormais à celle d'autres maîtres, mais en celui qui cherche à s'immiscer et à poursuivre quelque chose qui est déjà là, un discours qui court et qui doit être le moins personnalisé possible: «Dans le discours qu'aujourd'hui je dois tenir [...] j'aurais voulu me glisser subrepticement » (p.7);

- enfin, à propos des deux textes de Kant: «Qu'est-ce que l'esprit des lumières?» et «Qu'est-ce que la Révolution (française)?», Foucault formule une de ses grandes préoccupations: comment penser notre actualité? Il relève que, face aux événements majeurs de son époque, Kant ne se positionne pas de façon frontale comme on le faisait habituellement, en jugeant en termes d'autorité au nom d'un passé ou en vue d'un avenir. Il cherche au contraire un positionnement différent, non normatif, qui le conduit à poser, pour la première fois, l'actualité comme un problème à résoudre.

Foucault philosophe a souvent eu un tel rapport de biais avec d'autres disciplines, avec d'autres pratiques culturelles. Il entretient ces rapports, faits autant d'affinités que de tactiques, pour déplacer sa propre pensée et caractériser les déplacements de celle des autres. Une de ses premières préoccupations était d'ouvrir la philosophie en ajoutant quelques branches à l'alternative formée au début des années soixante par le marxisme d'un côté et la phénoménologie de l'autre. 
Il utilisa ainsi les cuvres littéraires et picturales (Roussel, Klossovski, Borgès; Bosch, Vélasquez, Magritte) pour introduire des questionnements nouveaux, des éclairages différents. C'est un rapport du même type qu'il entretint avec les sciences humaines. Il n'a jamais voulu - et encore moins prétendu -- être psychologue, historien ou politologue. Il eut des relations très décalées avec ces disciplines, les utilisant comme des terrains, y trouvant des chantiers nouveaux pour le philosophe et des prismes pour ouvrager sa propre pensée. Sur eux, il travailla toujours d'autres objets, formula toujours d'autres questions que ceux des spécialistes du domaine.

Dans Impossible prison, à propos de l'opposition entre l'histoire et la philosophie, Foucault répond à J. Léonard en refusant le clivage qui lui est présenté entre «la tâche sombre de l'exactitude», versus «la grande bousculade des idées approximatives» :

«Plutôt que de jouer pour la millième fois sur ce stéréotype, ne vaut-il pas mieux débattre sur les modalités, les limites et les exigences propres à deux manières de faire?

- l'une qui consiste à se donner un objet et à essayer de résoudre les problèmes qu'il peut poser;

- l'autre qui consiste à traiter un problème et à déterminer à partir de là le domaine d'objets qu'il faut parcourir pour le résoudre.» (p. 32)

Foucault utilise les mots et les questionnements des historiens pour en faire autre chose: le dépassement de la mort comme absolu dans Naissance de la clinique, la territorialisation de la raison dans Histoire de la folie, l'anthropologie comme moment temporaire de la pensée dans Les mots et les choses, la formation du pouvoir disciplinaire dans Surveiller et punir, etc.

Cette pratique d'ouverture de la pensée l'a conduit à poser empiriquement quatre principes épistémologiques.

\subsection{Quatre principes épistémologiques}

\section{a) Rompre avec le cercle anthropologique}

La rupture avec le "cercle anthropologique» est la voie que Michel Foucault propose dans les années soixante pour renouveler la philosophie. Son projet est de dépasser la perspective anthropologique qui n'en finit pas de tenter de répondre à la question «qu'est-ce que l'Homme?» et de tout expliquer en passant ou en revenant à l'homme.

L'humain est une catégorie de pensée marquée au sceau de l'histoire, elle est une production et non une essence. Il s'agit d'une catégorie temporaire. On a pu penser le monde sans y recourir. Le concept actuel d'homme 
est un moment dans le savoir, un palier de connaissance franchissable. Sa critique de la prégnance anthropologique est présente dès Naissance de la clinique, où Foucault souligne l'importance de l'expérience médicale dans le mouvement par lequel l'homme a pris une connaissance positive de lui-même. L'importance de la médecine dans la constitution des sciences humaines n'est pas seulement méthodologique, elle est aussi ontologique «dans la mesure où elle concerne l'être de l'homme comme objet de savoir positif». Cette critique s'affine dans la conclusion de l'ouvrage lorsqu'il s'interroge sur la possibilité pour l'individu d'être à la fois sujet et objet de sa propre connaissance (pp. 199-201).

Dans Histoire de la folie, le point de vue critique est plus affirmé encore. La conclusion du livre, intitulée «Le cercle anthropologique», est tout entière consacrée à cet accaparement par l'humain de toutes les expériences. L'insensé de l'âge classique est devenu l'aliéné, celui qui était au dehors s'est vu absorbé, dans la mesure où il renvoie à l'homme une part de sa vérité cachée. La représentation anthropologique de l'univers et de toutes les expériences vécues a tout absorbé, y compris ce qui lui était le plus étranger.

Mais ce n'est pas parce que cette catégorie totalisante a imposé son impérialisme qu'il faut lui attribuer des vertus analytiques absolues. Bien au contraire. Foucault considère qu'il est impossible de philosopher à partir d'une catégorie de pensée que l'on sait fragile, mieux mortelle. Or, dit-il :

Et voilà qu'en ce pli la philosophie s'est endormie d'un sommeil nouveau: non plus celui du dogmatisme mais celui de l'anthropologie. Toute connaissance empirique, pourvu qu'elle concerne l'homme, vaut comme champ philosophique possible où doit se découvrir le fondement de la connaissance, la clef de ses limites et finalement la vérité de toute vérité (Les mots et les choses, p. 352).

Ceci a conduit à la centration sur tout ce qui peut se donner en général à l'expérience de l'Homme.

«Pour réveiller un tel sommeil [...] il n'y a pas d'autre moyen que de détruire jusqu'en ses fondements le quadrilatère anthropologique. » (Les mots et les choses, p. 353)

Son projet s'énonce alors ainsi : «formaliser sans anthropologiser" (Les mots et les choses, p. 359).

Ce refus de l'approche anthropologique lui est beaucoup reproché par Habermas, en particulier dans «Les sciences humaines démarquées par la 
critique de la raison", chapitre du Discours philosophique de la modernité. Pour Habermas, les sciences humaines selon Foucault ne sont pas dégagées de leur origine médicale, clinique, c'est-à-dire :

- un savoir comptable et statistique sur le corps et la vie de l'Homme;

- les sciences humaines ne peuvent, d'après leur forme même, représenter qu'un amalgame de pouvoir et de savoir qui se renforcent mutuellement;

- les sciences humaines ne sont qu'une forme rationnelle et occultée des procédures d'examen.

Pour Habermas, Foucault occulte pour les besoins de sa démonstration certains acquis liés à l'existence de cette centration sur l'Homme, en particulier «l'État de droit» (p.345). Il lui reproche aussi de ne présenter aucune alternative. Il fait un parallèle avec Marcuse qui avait déjà dénoncé le jeu à double face d'une libération sexuelle contrôlée, mise en scène et commercialisée, débouchant sur une "désublimation répressive», "seulement, une telle analyse laissait ouverte la perspective d'une désublimation libératrice».

Chez Foucault, Habermas relève "l'occultation spécifique de tous les aspects qui ont permis d'appréhender l'érotisation et l'intériorisation de la nature subjective comme une avancée positive à la fois dans le domaine des libertés et dans celui des possibilités d'expression».

Il y a là une différence de positionnement radicale entre celui qui ne voit dans le savoir que des événements de vérité temporaires et celui qui y voit un moyen de libération.

Plus fondamentalement se pose entre eux la question des «universaux». Le refus des universaux de la pensée est une autre constante épistémologique de Michel Foucault.

\section{b) Refus des universaux de la pensée}

Un autre principe appliqué par Foucault est le refus de toute universalisation, de tout discours englobant. Il met ainsi à distance «l'esprit des Lumières ", c'est-à-dire la centralité de l'bumain, le critère universel de la raison et la croyance au progrès en tant que fondement incontournable de toute pensée. Tout comme il veut arracher la réflexion au cercle anthropologique, de même il veut repousser le critère absolu de la raison et se tenir à l'écart des jugements en termes de progrès-décadence.

- Il ne parle jamais par généralité, toujours au cas par cas. 
- Il repousse sans cesse ce qui pourrait laisser croire à de l'universel.

- Toujours il distingue, il particularise, et, s'il compare, c'est pour mettre à jour des différences de forme et non pour juger en termes positifs ou négatifs.

Michel Foucault est un casuiste, un intellectuel spécifique qui va jusqu'à refuser de théoriser explicitement sa propre démarche. Il travaille des questions spécifiques sur des terrains particuliers, toujours précisément situés dans le temps et l'espace. Il incite donc à la plus grande méfiance vis-à-vis des placages, des parachutes interprétatifs et des pronostics.

c) Les deux principes précédents sont des principes négatifs, le troisième est au contraire positif. Ce que Michel Foucault place au centre de sa recherche, ce sont «les Régimes de Vérité" ou «les Politiques de Vérité». Il entend par là que chaque formation sociale crée et est aussi créé par un «Régime de Vérité», c'est-à-dire par des discours qu'elle accueille et fait fonctionner comme vrais.

De là l'importance qu'il accorde à la formation des savoirs et aux systèmes de savoir qui en règlent les pratiques. Car ce sont eux qui fournissent les critères d'énonciation et de diffusion de ce qui est reçu, à un moment, comme vrai. Quand Foucault parle de «Régime de Vérité», il ne cherche pas à participer à la lutte entre théories. Il interroge plutôt les théories elles-mêmes sur leur système de vérité implicite. Il essaye de montrer chaque fois les points qu'elles ont mis en visibilité ainsi que les modes d'énonciation qui les ont accompagnées et qui forment la rationalité du discours, c'est-à-dire ses principes de découpage et d'organisation.

Ce troisième principe est repris de la philosophie des sciences (condition de possibilité de la science). Tout phénomène réel ou imaginaire n'existe, ne prend forme qu'au travers des catégories à partir desquelles, au travers desquelles on le pense: la réflexion critique passe donc d'abord par une histoire des concepts.

Parler d'histoire des concepts comme méthodologie d'une histoire des systèmes de vérité conduit aussi à envisager le changement. Comment passe-t-on d'un dispositif de visibilité et d'énonciation à un autre? Comment meurent les représentations? Foucault travaille aussi sur la différenciation entre Régimes de Vérité, il travaille sur les continuités et sur les discontinuités, sur l'entrelacement de ce qui s'efface (l'Archive) et de ce qui est en train d'advenir (l'Actualité). 


\section{d) Un matérialiste critique}

L'étude minutieuse des pratiques tant discursives que matérielles est le point par lequel Foucault est venu ajouter a l'histoire des sciences et des concepts un apport original. Le savoir sur le monde ne se cantonne pas au plan idéel, il provient aussi des épreuves et des découvertes de la pratique. L'histoire des sciences et de la philosophie à travers lesquelles il s'est formé cantonnait ses observations dans le registre des idées abstraites. Avec Naissance de la clinique et Histoire de la folie, Foucault trouve sa différence d'approche. Il montre comment de nouvelles pratiques, telles la dissection et l'autopsie, ont renouvelé les formes des connaissances médicales et psychiatriques, ont renouvelé la pensée médicale; ou comment l'enfermement asilaire a rendu la psychiatrie.

Dans ce sens, Foucault est un matérialiste qui ne s'attache pas aux concepts en soi, mais les envisage toujours au travers de leur inscription dans des actions concrètes, les saisissant lors de leur matérialisation. Naissance de la clinique est, selon ses mots, «l'essai d'une méthode dans le domaine si confus [...] de l'histoire des idées» (p. 197). Cette histoire s'appuie essentiellement sur le renouvellement de l'expérience clinique, de ses conditions matérielles et de sa théorisation, elle retrace l'émergence de nouveaux modes de visibilité et d'objectivation.

Il en va de même avec Histoire de la folie qui saisit la transformation des pratiques asilaires comme «une structure nouvelle d'expérimentation» renouvelant les représentations de la folie et suscitant le savoir psychiatrique.

Après la parenthèse, essentiellement conceptuelle, de Les mots et les choses et de L'archéologie du savoir, Surveiller et punir marque la reprise de cette méthodologie qui tire d'une analyse des pratiques ses propositions principales pour une histoire des idées. C'est du renouvellement des technologies de surveillance et d'emprise concrète sur les corps que Foucault extraira sa théorie du pouvoir disciplinaire.

De ce rapport éminemment critique aux sciences de l'homme, Foucault a donc tiré un certain nombre de règles épistémologiques qui font l'originalité de sa position intellectuelle. Comment alors utiliser son cuvre? Comme une simple «boîte à outils»? C'est ce qu'il avança un jour :

Tous mes livres sont de petites boîtes à outils [...] Un livre est fait pour servir à des usages non définis par celui qui l'a écrit. Plus il y aura d'usages nouveaux possibles, plus je serai heureux.

Il y a chez Foucault au moins trois sortes de boîtes à outils: 
1. boîte à outils conceptuels ;

2. boîte à méthodes:

- pour le choix des terrains liés à l'actualité, à la question centrale «qui sommes-nous en train de devenir?»; énoncés;

- pour la méthode d'analyse du discours et la priorité donnée aux

3. bô̂te à outils épistémologiques :

- refus de l'anthropomorphisme;

- refus des universaux;

- analyse prioritaire des concepts, des outils de la connaissance;

- priorité à l'analyse des pratiques comme point de départ.

Comment ces principes peuvent-ils s'opérationnaliser sur un secteur spécialisé comme la sociologie du droit?

\section{LA DIMENSION JURIDIQUE DU POUVOIR}

Parmi tous les discours de vérité, ceux tenus par le droit - et mieux ceux qu'on lui fait tenir - tiennent depuis deux siècles une place croissante. Aux vérités produites par les croyances théologiques puis par l'autorité arbitraire des souverains ont fait place d'autres énoncés de vérité qui adoptent le plus souvent une forme juridique. L'œuvre de Foucault peut nous aider à préciser comment s'énoncent ces "vérités légales", selon quelle matérialité et selon quels dispositifs elles s'organisent. Cette approche donne, au moins, deux grandes questions de travail sur le droit:

- il ne peul s'agir de se lancer dans une recherche de l'«essence» ou de la «nature» du droit, mais de prendre les énoncés juridiques comme une fiction expressive et organisatrice. On se demandera alors à quel type de pratique et de pensée spécifiques correspond telle forme juridique et quels en sont les effets.

- Foucault invite aussi à penser le changement dans les dispositifs juridiques. Le droit ne l'intéresse pas directement, il l'utilise comme un indicateur des systèmes de pouvoir. Quelle relation existe-t-il alors entre telle rationalité politique et telle forme juridique et comment les transformations de l'une se répercutent-elles sur l'autre? 
Une approche de sociologie du droit peut se référer à la théorie du pouvoir de Foucault telle qu'elle s'exprime par exemple dans son texte «Pourquoi étudier le pouvoir: la question du sujet» (in Dreyfus et Rabinow, Foucault, un parcours philosophique) ou dans ses textes sur «la gouvernementalité».

\subsection{Relations de pouvoir et objectivation du sujet}

Foucault n'est pas un théoricien du pouvoir, du moins il ne l'est pas directement. Une de ses préoccupations constantes a été celle de la production du sujet. Le «sujet» n'est pas pour lui un donné ahistorique, une entité préconstituée et constante, il n'est pas un irréductible.

Pour reprendre une expression de Deleuze, le sujet est chez Foucault «une forme, résultat d'un rapport de force ${ }^{2}$ ». Le sujet est le produit d'un jeu de tensions entre des procédures d'objectivation et de subjectivation. C'est pourquoi Foucault accorde une telle importance aux savoirs et aux pouvoirs dans la mesure où ce sont eux qui règlent l'essentiel de ces procédures. Ce n'est donc pas le pouvoir en soi qui intéresse Foucault, mais les rapports de pouvoir et de savoir, c'est-à-dire les dispositifs qui produisent des identités (malade/homme sain, aliéné/homme de raison, délinquant/ citoyen normé, sujet moral/pervers) et des relations entre sujets (rapport médical, psychiatrique, policier, carcéral, thérapeutique, disciplinaire...). Ces dispositifs où s'entremêlent savoirs et pouvoirs connaissent des transformations dans la mesure où ils sont l'objet et le lieu de rapports de force.

Foucault a introduit des apports essentiels aux analyses en termes de pouvoir, d'une part en les déglobalisant, d'autre part en les vitalisant.

- Il s'est tout d'abord démarqué des approches globalisantes qui, jusque-là, prévalaient en se refusant à prendre «le pouvoir» comme un tout homogène et stable dont on chercherait l'origine pour la légitimer ou la contester. Ainsi, contrairement aux approches de l'histoire politique classique ou, à l'opposé, au marxisme, Foucault a désétatisé l'analyse du pouvoir. Il s'est refusé à faire de l'État la source de tous les pouvoirs, le foyer qui diffuse des ordres et qui ne cesse de se renforcer. Il propose de travailler sur le «comment des pouvoirs», sur l'analyse des différents types de relations de pouvoir. Avec lui, on est passé du «pouvoir» à la question des «modalités du pouvoir».

2. G. DELEUZE, Foucault, «Sur la mort de l'homme», Paris, Éditions de Minuit, 1986, p. 181. 
- D'autre part, les relations de pouvoir ne sont pas unilatérales, elles ne s'exercent pas sous la seule forme du commandement, de l'impératif. Il y a des rapports de pouvoir interactifs. D' où l'importance qu'il accorde aux relations de pouvoir ascendantes (et pas seulement descendantes) ainsi qu'aux résistances, aux oppositions et aux conflits. Il n'envisage pas le pouvoir du seul point de vue de sa rationalité interne, mais aussi sous l'angle de l'affrontement de stratégies différentes de pouvoir.

Dans son analyse des formes contemporaines de pouvoir, qu'il nomme "bio-pouvoir», Foucault insiste sur la notion de normes. Cette forme de pouvoir qui s'exerce sur les sujets ne repose pas sur l'interdit ou la sanction, mais sur la diffusion généralisée de «disciplines», c'est-à-dire de normes de perception et de comportement intériorisées par les sujets en tant que facteurs de développement et créatrices d'effets positifs: normes sanitaires, de production, de sécurité, de plaisir, etc.

Enfin, on peut dire que Foucault a toujours combiné deux approches globale et analytique - du pouvoir qui dépassent la simple somme des besoins individuels. Il envisage d'une part des rapports de pouvoir globaux, ceux qui concernent les populations considérées comme des ensembles à gérer selon des besoins spécifiques. D'autre part, il s'attache à des rapports de pouvoirs spécifiques interindividuels. Chacun de ces points de vue est lié à des formes d'objectivation des sujets par des pratiques concrètes et des savoirs. L'économie, la démographie, l'histoire, l'hygiène publique d'un côté; la médecine, la psychologie, la pédagogie, la sexologie de l'autre.

Cette combinaison de deux plans d'analyse est une constante dans sa méthode depuis Naissance de la clinique jusqu'à Histoire de la sexualité:

- double objectivation de la maladie (physique puis mentale), en tant que problème global devant donner lieu à une administration (gouvernement des épidémies et des insensés) et en tant qu'enjeu dans la relation malade-soignant (relation clinique et thérapeutique);

- double objectivation de la délinquance, en tant qu'ensemble de conduites irrégulières donnant lieu à un traitement différentiel et en tant que lieu d'expérimentation des disciplines;

- double objectivation de tous les sujets par le pouvoir pastoral, source des formes contemporaines de «bio-pouvoir». Gouverner les populations, c'est, d'une part, les conduire en tant qu'ensemble vers un bien, vers un salut, vers un devenir positif; c'est aussi s'appuyer pour cela sur une incitation au «gouvernement de soi» par chacun, sur l'intériorisation d'une autodiscipline de chacun et des rapports interindividuels. 
L'analyse du pouvoir selon Foucault articule donc ces deux niveaux d'objectivation :

- celui du «gouvernement des populations», à savoir l'ensemble des techniques d'orientation et de contrainte des comportements collectifs;

- celui du «gouvernement des sujets», à savoir les relations où s’impliquent des sujets actifs et non de simples robots se conformant à des directives unilatérales.

C'est en m'appuyant sur cette double approche que j'ai mené un certain nombre de travaux de sociologie juridique. On peut en effet parler également d'une double objectivation juridique :

- dans la mesure, tout d'abord, où le droit est un instrument majeur de gouvernement des populations:

- dans la mesure, ensuite, où les rapports de pouvoir interindividuels sont aussi une dimension juridique non négligeable où s'affrontent des identités d'action et se développent des interactions juridiques.

\subsection{La place du droit dans la gouvernementalité}

C'est à l'occasion de ses cours de l'année 1977-1978 sur le thème «Sécurité, territoire et population» que Foucault explicita sa critique des modèles d'interprétation étatistes et énonça sa conception de la gouvernementalit $e^{3}$. Contrairement à la théorie politique classique, qui distingue toujours les sociétés selon leur régime politique, il avança une distinction des types d'État (État de justice, État d'administration, État de police). Et cette distinction se fonde non pas sur des critères idéologiques, mais sur des critères de pratiques, sur ce que B. Barret-Kriegel nomme «la consistance du politique ${ }^{4}$ », la matérialité de ses actions. Pour Foucault, l'État (auquel il mettrait volontiers une minuscule) n'a jamais eu «cette unité, cette individualité, cette fonctionnalité rigoureuse qu'on lui prête souvent». Il y voit plutôt une «réalité composite» qui a acquis une force particulière avec la généralisation à partir du XVIII ${ }^{\mathrm{e}}$ siècle de techniques de gouvemement. «Peut-être ce qu'il y a de plus important dans notre modernité, c'est-à-dire pour notre actualité, ce n'est pas l'étatisation de la société, c'est ce que j'appellerais plutôt “la gouvernementalisation" de l'État.» Foucault conduit ainsi à s'attacher davantage au «comment» du pouvoir politique qu'à son «essence», à son «idéologie», à ses «infrastructures».

3. M. FOUCAULT, De la gouvernementalité, Paris, Éditions du Seuil, 1989.

4. B. BARRET-KRIEGEL, L'État et les esclaves, Paris, Calmann-Lévy, 1977. 
L'État n'est pas simplement un ensemble d'instruments de coercition et de répression; il existe aussi à travers des instruments de production normative, orientant les pratiques collectives et individuelles, normant les comportements, instituant des règles de droit. C'est l'accent mis sur cette positivité des pratiques de gouvernement qui fait l'originalité de l'apport de Foucault.

À partir de là, et pour se limiter aux travaux portant spécifiquement sur les «Régimes de Vérité juridique», on peut distinguer plusieurs pistes possibles.

- Tout d'abord les travaux de B. Barret-Kriegel qui portent sur les tensions existantes entre «État de droit» et «État de police», entre un État instrument de garantie des droits et des libertés individuelles et un État instrument de sûreté et de protection des populations. Le premier est, encore aujourd'hui, au mieux programmatique, au pire un simple habillage du second qui s'est imposé depuis la fin du XVIII ${ }^{\mathrm{e}}$ siècle et menace toujours de l'intérieur les régimes à prétention démocratique.

- Dans son ouvrage multidimensionnel sur «L'État Providence», F. Ewald s'attache à caractériser ce que l'on pourrait appeler une deuxième phase du pouvoir disciplinaire ou un deụxième âge du bio-pouvoir, celui qui est marqué par la montée d'une nouvelle rationalité politique, l'assurancialisation ${ }^{5}$. L'autoguidage des comportements tend à se transformer en passant d'un système juridique de pleine responsabilité à un système de responsabilité diluée par la socialisation des risques induite par les assurances. Le besoin de sûreté générale tend à convertir tout danger en risque susceptible d'être garanti. Les obligations du contrat social font place aux revendications de solidarité et à la crispation des demandes corporatistes. Et aux principes universaux du droit de la Révolution et de l'Empire succède un droit gestionnaire de changements permanents.

- Pour ma part, et essentiellement à travers des questions de droit pénal, je me suis intéressé au pluralisme et à la diversification des instruments juridiques. Il s'agit moins de dénoncer leurs incohérences ou de relever leur hétérogénéité que de montrer les effets de tension à l'intérieur du système juridique, dans la mesure où ces tensions sont des facteurs essentiels de changement. Au sein d'une formation sociale, à un moment historique donné, on voit coexister des formes juridiques dominantes (le

5. F. EWALD, L'État Providence, Livre III, «Assurance sociale», et Livre IV, «L'ordre normatif », Paris, Grasset, 1986. 
«sujet de droit», des peines rétributives, la notion de «service public») et des formes dominées. Ces dernières désignent les lignes de fracture des dispositifs juridiques et sont des indicateurs de changement.

À l'observation, on peut en distinguer de deux sortes :

- des survivances d'anciennes rationalités juridiques en voie d'extinction, mais parfois aussi bien vivantes (droit rural et forestier coutumier, droit des commerçants, pratiques transactionnelles);

- et des émergences de nouveaux rapports juridiques (droit pénal restitutif, règles incitatives, assurances, etc.).

On est ainsi conduit à se tenir à l'écart des interprétations évolutionnistes et à s'attacher à la complexité des modèles combinant des formes juridiques et s'appuyant sur des rationalités légitimantes diverses et inscrites dans une dynamique de changement.

\subsection{Rapports juridiques et rapports de pouvoir}

On peut aussi envisager à un niveau micro-analytique la question des rapports juridiques. Là encore, Foucault induit une critique des conceptions dominantes qui n'envisagent le droit qu'en termes de commandement sanctionné. À l'observation, la place du droit dans les rapports sociaux ne se traduit pas par la simple soumission ou par la résistance à des «diktats», mais par des jeux de relations entre tel acheteur et tel vendeur, tel administré et telle administration, tel salarié et tel employeur. Le droit apparaît avant tout comme une ressource pour l'action. Il ne s'agit pas d'un instrument de pouvoir qui s'impose unilatéralement d'un sommet tout-puissant à une base passive. Le droit n'existe qu'à partir du moment où il est mobilisé pour réguler des situations toujours particulières.

La perspective dessinée par Foucault, qui se propose de «formaliser sans anthropologiser », a également en droit une pertinence particulière. Le droit ne se résume pas au sujet de droit, il crée des identités d'action qui constituent des ressources mobilisables pour chaque acteur. Cette mobilisation s'effectue dans des contextes, en fonction de situations qui n'ont pas une nature juridique préexistante, celle-ci sera construite dans les interactions. La qualification tout comme le choix des voies de règlement s'accomplissent dans ces relations toujours mobiles. Chaque acteur n'est pas réductible à une identité juridique, c'est le contexte qui la détermine.

En matière juridique, être en situation de pouvoir, c'est avoir et garder la mâtrise des règles applicables dans les processus où l'on se trouve 
impliqué. Inversement, être en position dominé, c'est se voir imposer une identité d'action, demeurer sans prise sur le déroulement des interactions. Le droit n'est donc pas par essence "garantie des libertés fondamentales» ou «instrument de domination ». Il reste un enjeu dans les relations entre acteurs, chacun en mobilisant certains aspects selon des ressources contextuelles.

En conclusion, et comme ligne de fuite de cette analyse des rapports de pouvoir, je proposerai une confrontation possible entre l'approche de M. Foucault et celle de M. Weber. Nietzsche a été un de leurs inspirateurs communs, en particulier lorsqu'il s' agit de souligner le caractère historique, contingent des critères de vérité. À la limite, pour eux, il n'y a pas de faits, pas de vérité objective, il n'y a que des interprétations, des regards. D'autre part, Foucault et Weber se retrouvent dans une position intellectuelle qui les tient à distance de l'exercice direct du pouvoir politique. Comment articuler la philosophie avec l'actualité et ses urgences sans céder à la tentation de la prophétie? De plus, l'un et l'autre ont accordé dans leurs analyses une place importante au développement de la rationalisation du monde et à ses effets sur les techniques d'objectivation des sujets. Ils ont aussi souligné la coexistence au sein des sociétés de principes de rationalisation différents et les tensions qui en découlent. Cette approche globalisante est aussi complétée, chez l'un comme chez l'autre, par une approche des formes de subjectivation des sujets; le gouvernement des sujets par eux-mêmes chez Foucault, la question de l'éthique et de l'orientation des conduites chez Weber. Enfin, aux théories holistes qui ont longtemps dominé la philosophie et l'histoire, leurs méthodes et leurs modélisations se retrouvent dans le rôle déterminant qu'ils donnent aux activités sociales en opposition avec tous les déterminismes. 